\title{
Analysis of 14 genetic polymorphisms for risk factors in Malaysian essential hypertension with or without type 2 diabetes mellitus subjects
}

\begin{abstract}
Objectives The objective of this study was to determine the association of insertion/deletion (I/D), G2350A and T3892C polymorphisms of angiotensin converting enzyme (ACE) gene, $\mathrm{A} 20 \mathrm{C}$ and A6G polymorphism of angiotensinogen (AGT) gene, BglI and MboI polymorphisms of renin gene, I/D of alpha 2B adrenoceptor gene, Gly460Trp of aldosterone synthase gene, Lys173Arg of adducin gene, A6244G polymorphism of the insulin receptor gene, S477X polymorphism of the lipoprotein lipase gene, C511T polymorphism of the interleukin gene and C825T polymorphism of the $\mathrm{G}$ protein beta 3 subunit (GN $\beta 3$ ) gene in essential hypertension (EHT) with or without type 2 diabetes mellitus (T2DM) of Malaysian subjects. Methods This cross-sectional study includes 70 EHT without T2DM, 65 EHT with T2DM and 75 unrelated healthy control subjects. Genomic DNA was extracted from the peripheral blood. The polymerase chain reaction (PCR)-restriction fragment length polymorphism, mutagenically PCR and the hot-start PCR methods were carried out to detect the genotypes of the various genetic polymorphisms. All the PCR products and the restricted fragments were resolved electrophoretically on agarose and polyacrylamide gels. Statistical analyses was done using SPPS version 14.0. Results Among the 14 genetic polymorphisms, only I/D and G2350A polymorphism of ACE gene, I/D polymorphism of alpha adrenoceptor gene and A6G variant of AGT gene differed significantly $(\mathrm{p}<0.05)$, while other polymorphisms did not differ significantly $(p>0.05)$ when compared to control subjects. Conclusions The alleles of I/D and G2350A polymorphisms of ACE, A6G of AGT and I/D of alpha $2 \mathrm{~B}$ adrenoceptor genes can be considered as possible genetic markers or predisposing risk factors for EHT with or without T2DM in Malaysian subjects.
\end{abstract}

Keyword: Genetic polymorphism; Essential hypertension; Type 2 diabetes mellitus; Malaysia; Angiotensin converting enzyme 